\title{
Estimation of blood pressure parameters using ex-Gaussian model
}

\author{
Artur Poliński \\ Gdansk University of Technology \\ in Gdańsk \\ Narutowicza 11/12, 80-233 Gdańsk, Poland \\ Email: apoli@eti.pg.gda.pl
}

\author{
Tomasz Kocejko \\ Gdansk University of Technology \\ in Gdańsk \\ Narutowicza 11/12, 80-233 Gdańsk, Poland \\ Email: tomasz.kocejko@pg.gda.pl
}

\begin{abstract}
The paper presents an example of model-based estimation of blood pressure parameters (onset, systolic and diastolic pressure) from continuous measurements. First, the signal was low pass filtered and its quality was estimated. Good quality periods were divided into beats using an electrocardiogram. Next, the beginning of each beat of the blood pressure signal was approximated basing on the function created from the sum of two independent distributions: Gaussian and exponential. The nonlinear least square method was used to fit measurement data to the model. The initial conditions for the fitting procedure were selected for each beat on the basis of its parameters. Finally, the diastolic and systolic values of blood pressure and onset were determined.
\end{abstract}

\section{INTRODUCTION}

$\mathbf{T}$ HE BLOOD pressure analysis allows for a certain characterization of the cardiovascular system and thus the patient state. Therefore, it is very important to accurately estimate its characteristic values from noisy data (measurements). The methodology requires that certain steps are performed. First, the signal quality should be estimated in order to skip the fragments of the data which are too noisy. This can be done by using the methods proposed in [1], [2]. Next, the characteristic points of a full beat of blood pressure signal should be estimated. A different method of estimation can be utilized including a windowed and weighted slope sum function [3], a filter bank with variable cutoff frequencies, rank-order nonlinear filters, and decision logic [4], inflection and zero-crossing points of blood pressure, and then combinatorial amplitude and interval criteria to select the onset and systolic peak [5], wavelets [6], principal components [7], waveform descriptor compared with a customized template [8], determined lines and polynomial approximation [9] or Fourier series interpolation [10].

In our studies we have decided to use a model-based approach to obtain signal parameters. In such an approach the approximation results correspond to the selected model. Therefore, it is important that the model has a similar shape to the real signal. A simple solution is to use a polynomial model

This work was partially supported by European Regional Development Fund concerning the project: UDA-POIG.01.03.01-22-139/09-00 -"Home assistance for elders and disabled - DOMESTIC", Innovative Economy 20072013, National Cohesion Strategy and by Statutory Funds of Electronics, Telecommunications and Informatics Faculty, Gdansk University of Technology

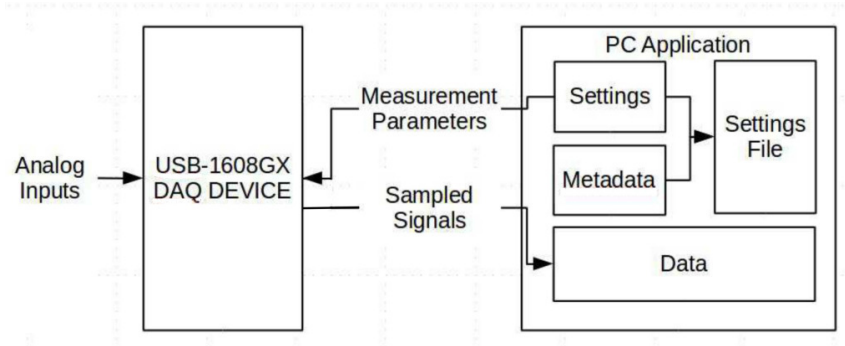

Fig. 1. Communication between DAQ and data receiving software

(like in R-wave estimation [11], which does not guarantee, however, correct results for longer parts of a signal. We have decided to use the more complicated exponentially modified Gaussian function. The exponentially modified Gaussian function was used in different applications like chromatography [12] or cell proliferation and differentiation [13], , but to our knowledge, it has not been used in blood pressure modelling. The practical limitation of such a simple model is that the pressure waveform is a combination of incident and reflected waves. It affects the estimation of systolic pressure. In such an approach, the estimation of model parameters is crucial. Since there is a nonlinear dependence of the model parameters on measurement data, the nonlinear least square approach was used. The model parameters obtained allow to extract diastolic and systolic pressure, but also to compare different pulses using reconstructed parameters. These parameters allow a further data analysis including the dependence between heart rate and blood pressure analysis [14] or estimation of patient condition [15], [16].

\section{MATERIAL AND Method}

The continuous blood pressure and ECG were measured using a custom ECG module and CNAP monitor. Both devices were connected to the 16-channel USB Data Acquisition DAQ Module. All data relayed by the DAQ module were recorded by custom software. The block diagram (Fig. 1) presents a general overview of communication between DAQ and the proposed custom recording software.

The rapid measurement and analysis of the ECG signal was enabled by the dedicated ECG optimized pre-amplifier de- 


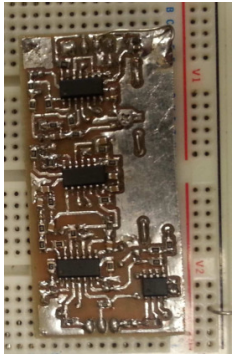

Fig. 2. The prototype of the custom ECG measuring module

signed and manufactured for the purpose of this study (Fig. 2). The overall amplification of the unit was set to approximately 5000. The bandwidth was limited to $0.05 \mathrm{~Hz}-60 \mathrm{~Hz}$ with a dedicated $50 \mathrm{~Hz}$ notch filter. A similar solution was used in [17] and [18]. To improve the CMRR, a dedicated DRL circuit was implemented. Blood pressure was measured by means of a standalone CNAP monitor. It enabled the continuous monitoring of blood pressure and pulse rate. All utilized hardware and software came from different vendors which made the integration of the measurements slightly challenging. The CNAP monitor analogue output was connected, next to the custom ECG unit, to the additional PC by means of the DAQ Module. Dedicated software was used for reading the data from the data logger. The measurements were triggered over the network by the UDP protocol. Because ECG and CNAP were registered by means a DAQ data logger there was no delay between measurements. The use of external trigger (over network) allows to extend the measurements by additional parameters like respiration rate, etc. The UDP protocol ensured simple and quick data transfer. Moreover, the UDP allowed for multicast traffic which is very convenient for synchronized measurements of different biosignals.

The data were collected from three healthy men at the age of 28, 33 and 47. The duration of measured signals (including blood pressure, ECG, respiration and eye movement - not used in the present study) was 120 seconds for each person.

The measured signals were sampled with $1 \mathrm{kHz}$ frequency. This relatively high frequency is required to obtain a large number of data for each beat of pressure. This allows to increase the accuracy of the approximation results. First, the continuous blood pressure signal was filtered using a low pass FIR filter (order 1256) with cut off frequency equal to $16 \mathrm{~Hz}$. This made it possible to remove the high frequency noise without modifying the shape of the signal and respiratory influence. The Matlab filtfilt function was used, and consequently there was no delay between the raw and filtered data. After blood pressure preprocessing, the R-wave of the electrocardiogram was detected using [19] algorithm. The shape of the blood pressure wave was good enough to skip the above-mentioned quality measure calculations. For each R-wave detected, the minimum of the blood pressure signal was sought. The search was carried out up to $200 \mathrm{~ms}$ from the R-wave detected. If there was more than one point of the minimum, than the latest minimum was chosen as a reference $\left(t_{\min }\right)$. Next, the time of the maximum of the blood pressure was sought at the $400 \mathrm{~ms}$ window. If there was more than one point, the latest value was chosen $\left(t_{\max }\right)$. The last step was choosing the time interval for signal approximation $\left(t_{\min }-10 \mathrm{~ms}, t_{\max }+150 \mathrm{~ms}\right)$. Each beat was approximated using the model derived from convolution of two independent additive processes: Gaussian and exponential (ex-Gaussian model)

$$
\begin{gathered}
f(t)=C+M \frac{\lambda}{2} \exp \left(\frac{\lambda\left(2 \mu+\lambda \sigma^{2}-2 t\right)}{2}\right) \times \\
\left(1-\operatorname{erf}\left(\frac{\mu+\lambda \sigma^{2}-t}{\sqrt{2} \sigma}\right)\right)
\end{gathered}
$$

where $M$ is a constant required to stretch the function to the desired range of beat pressure, $C$ is a constant which determines the level of the signal (diastolic blood pressure), $\mu$, $\sigma, \lambda$ are parameters describing the properties of the function ( $\mu$ and $\sigma$ come from the Gaussian model and $\lambda$ from the exponential model). The $\operatorname{erf}(\cdot)$ is an error function

$$
\operatorname{erf}(x)=\frac{2}{\pi} \int_{0}^{x} e^{-t^{2}} d t
$$

Having $t_{\min }$ and $t_{\max }$ initial values for fitting were calculated as follows

$$
\begin{aligned}
\mu_{\text {init }} & =t_{\max } \quad \sigma_{\text {init }}=\left(t_{\max }-t_{\min }\right) / 2 \quad \lambda_{\text {init }}=10 \\
M_{\text {init }} & =\left(B P\left(t_{\max }\right)-B P\left(t_{\min }\right)\right) / 4 \quad C_{\text {init }}=B P\left(t_{\min }\right)
\end{aligned}
$$

where $B P$ is the registered continuous blood pressure signal. The lsqnonlin Matlab function was used to find the global minimum for the nonlinear least square problem. The systolic and diastolic blood pressure was determined from the minimum and maximum values of each estimated beat. A more complicated issue is onset calculation. In the approach assumed, it was represented by the point of the maximum curvature of the estimated model, where the curvature was determined from

$$
\text { curve }=\frac{\left|f^{\prime \prime}(t)\right|}{\left(1+\left(f^{\prime}(t)\right)^{2}\right)^{3 / 2}}
$$

To allow comparison with other methods, the algorithm was tested on publicly available data. The 037 record from the MIMIC database [20] was used. Since the data were sampled at $125 \mathrm{~Hz}$, the FIR filter was redesigned (order 157). The analysed data were restricted to the beats with $\mathrm{SBP}<180 \mathrm{mmHg}$ and DBP $>20 \mathrm{mmHg}$. This simple operation was conducted to remove the extremely high and low peaks. The median error for SBP, DBP and onset as well as the RMS error (RMSE)

$$
R M S E=\sqrt{\frac{1}{N} \sum_{i=1}^{N}(\operatorname{data}(i)-\operatorname{approximation}(i))^{2}}
$$

were calculated for the first 15000 beats. In equation 5, data represents either SBP, DBP or onset obtained from annotations, while approximation represents the same parameters obtained from the algorithm proposed. The data values are integers so approximation values were convert integers as well. 


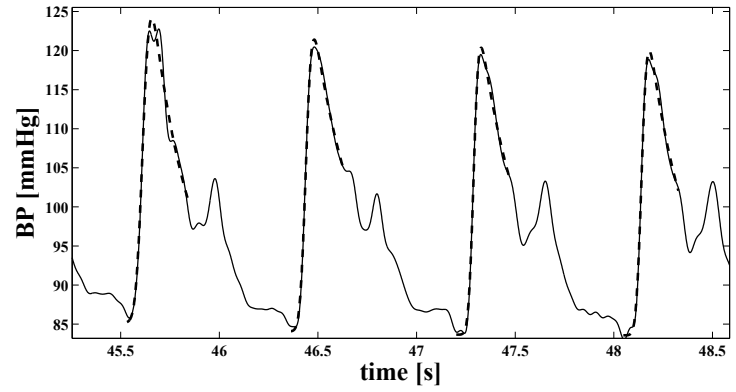

Fig. 3. Filtered signal and its approximation (dotted line)

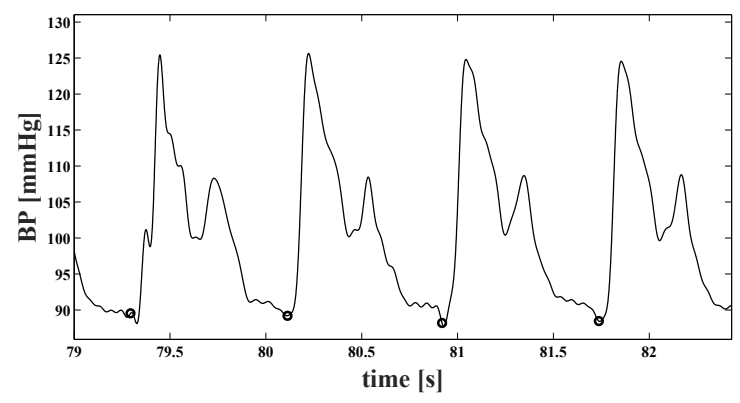

Fig. 4. An example of the onset points (circles)

\section{RESULTS}

Example of the fitting results (Fig. 3) and founded onset points (Fig. 4) as well as the influence of sampling frequency on DBP and SBP are presented (Figs. 5 - 6).

There were certain difficulties with the analysis of the reference data for 298 of 15000 beats. Modification of one initial data from $\sigma_{\text {init }}$ to $0.6 \sigma_{\text {init }}$ succeeded in 187 cases, while for the last 111 cases, further modification of one initial data from $0.6 \sigma_{\text {init }}$ to $0.5 \sigma_{\text {init }}$ succeeded in 26 cases. Convergence was not obtained for 85 beats. The median error for SBP, DBP and onset was equal to $2 \mathrm{mmHg}, 2 \mathrm{mmHg}$ and 0 samples, respectively, while the $R M S E$ error was equal to $4.97 \mathrm{mmHg}, 3.23 \mathrm{mmHg}$ and 2.40 samples. Removing $1 \%$ of the worst approximation results reduced the $R M S E$ error to

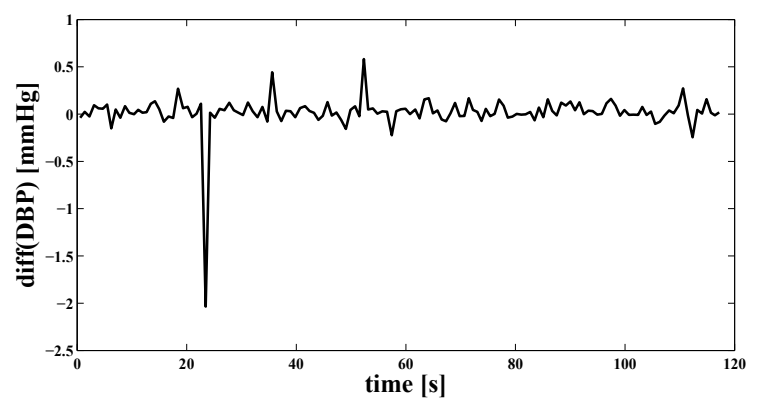

Fig. 5. The difference in estimated diastolic blood pressure (for $1 \mathrm{kHz}$ and $125 \mathrm{~Hz}$ sampling frequency)

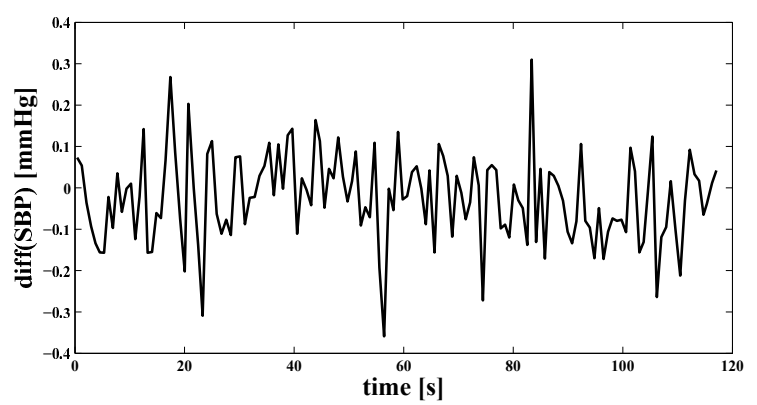

Fig. 6. The difference in estimated systolic blood pressure (for $1 \mathrm{kHz}$ and $125 \mathrm{~Hz}$ sampling frequency)

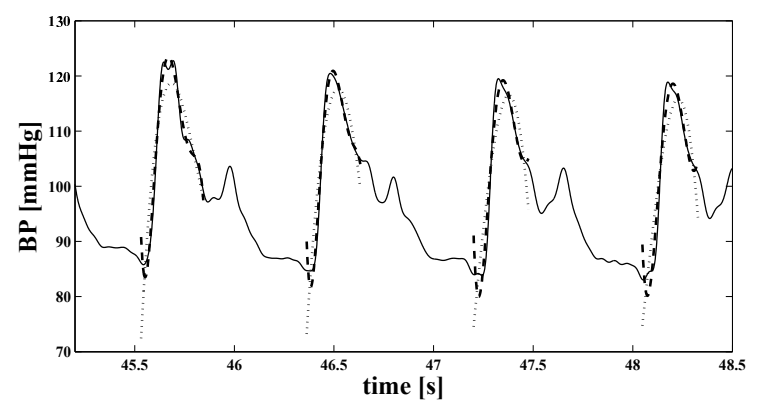

Fig. 7. Filtered signal and its polynomial approximation (dotted line - 3-rd, and dashed line 5-th degree)

$3.08 \mathrm{mmHg}, 2.64 \mathrm{mmHg}$ and 2.28 samples, respectively.

\section{Discussion AND CONClusions}

The results of approximation were highly satisfactory (Fig. 3 ). In the case of polynomial approximation, the results were much worse. An example of such approximation using 3-rd and 5-th degree polynomial is presented in Fig. 7. The difference between systolic and diastolic blood pressure calculated from low pass filtered data was similar to the approximated one. To reduce the problem of onset detection, the ECG signal was used as a reference. The main problem in nonlinear least square fitting is choosing the starting point for every iteration. Its influence was estimated by perturbing initial conditions. The results of fitting were most sensitive to the $\mu$ parameter. Even a change of $5 \%$ in the initial value caused poor fitting results. The distortions present in the recorded signal caused problems with the estimation of the fitting error. Thus, the results were compared to the fitting with the initial values. The quality of fitting using the initial values was estimated by visual inspection and a comparison of diastolic and systolic blood pressure with the values calculated for the low pass filtered blood pressure signal (defined as the minimum of blood pressure after R-wave - for the diastolic, and the maximum after DBP for the systolic). The fitting results were much less sensitive to the initial value of $\sigma$. Satisfactory results were obtained for values which were as much as ten times smaller and five times greater. Similar results were obtained for the initial $\lambda$ estimation. The method presented was more 
sensitive for the initial values of $C$ and $M$. Based on the experiment conducted we have assumed that a deviation of $5 \%$ is acceptable for $C$. In the case of parameter $M$, satisfactory results could be obtained when the value was in the range between $50 \%$ and $120 \%$ of the initial value.

The nonlinear least squares fitting can use the Jacobian calculated analytically or numerically. We did not notice any significant differences in the results obtained regarding the method of Jacobian calculation. The difference in systolic and diastolic values was lower than $10^{-3} \mathrm{mmHg}$, while the difference in onset was lower than $0.1 \mathrm{~ms}$.

The sampling frequency did not influence the results obtained. Down sampling of the blood pressure signal from 1 $\mathrm{kHz}$ to $125 \mathrm{~Hz}$ did not modify the estimation of DBP and SBP significantly (except for one sample) (Figs. 5 and 6).

The diastolic blood pressure can be easily obtained from the model $(\mathrm{DBP}=C)$. The analytic estimation of systolic blood pressure requires solving a nonlinear equation. The advantage of the proposed approach is that the calculated parameters: $\mu$, $\sigma, \lambda$ can be used to estimate the arteries' condition and their changes.

In our approach the onset estimation bases on finding the maximum curvature of the estimated model curve. To prevent finding the maximum value of the model as the point of maximum curvature, the search was limited to $50 \mathrm{~ms}$ after the beginning of each beat. The sampling frequency did not have a large influence on onset detection. The difference between values obtained for $1 \mathrm{kHz}$ and $125 \mathrm{~Hz}$ sampling frequency was lower than $7 \mathrm{~ms}$ (except for three peaks). The approach adopted for onset detection can be modified by introducing an additional parameter $p$, which will allow the onset point to be shifted. The curvature will then be defined as

$$
\frac{\left|f^{\prime \prime}(t)\right|}{\left(p+\left(f^{\prime}(t)\right)^{2}\right)^{3 / 2}} \text {. }
$$

In general, the reference ECG signal is not required. The beat localization can be obtained by using only the blood pressure waveform (for example by using the algorithm proposed in [3]), and the proposed model can be then used to obtain signal parameters.

The results obtained from the reference data are satisfactory. Some problems may be due to the relatively low sampling frequency and possible distortions in the analysed signal. Better results can be obtained by further modifying the initial parameters.

The analysis of all the results obtained enables the conclusion that the proposed method of blood pressure parameters estimation using the ex-Gaussian model is suitable to the requirements and reliable.

\section{REFERENCES}

[1] W. Zong, G. B. Moody, and R. G. Mark, "Reduction of false arterial blood pressure alarms using signal quality assessment and relationships between the electrocardiogram and arterial blood pressure", Med Biol. Eng. \& Comput., vol. 42, pp. 698-706 September 2004. doi $10.1007 / \mathrm{BF} 02347553$
[2] J. X. Sun, A. T. Reisner, R. G. Mark, "A signal abnormality index for arterial blood pressure waveforms", Computers in Cardiology, vol. 33 pp. 13-16, 2006

[3] W. Zong, T. Heldt, G. B. Moody, R. G. Mark, "An Open-source Algorithm to Detect Onset of Arterial Blood Pressure Pulses", Computers in Cardiology, vol. 30, pp. 259-262, 2003. doi:10.1109/CIC.2003.1291140

[4] M. Aboy, J. McNames, T. Thong, D. Tsunami, M. S. Ellenby, and B. Goldstein, "An Automatic Beat Detection Algorithm for Pressure Signals", Trans. on Biomed. Eng., vol. 52, no. 10, pp. 1662-1670 October 2005. doi: 10.1109/TBME.2005.855725

[5] B. N. Li, M. C. Dong, M. I. Vai, "On an automatic delineator for arterial blood pressure waveforms", Biomedical Signal Processing and Control, vol. 5, pp. 76-81, 2010. doi:10.1016/j.bspc.2009.06.002

[6] A. Pachauri and M. Bhuyan, "Wavelet Transform Based Arterial Blood Pressure Waveform Delineator", International Journal of Biology and Biomedical Engineering, Issue 1, vol. 6, pp. 15-25, 2012.

[7] P. Xu, M. Bergsneider, X. Hu, "Pulse onset detection using neighbor pulse-based signal enhancement", Med. Eng. \& Phys., vol. 31, pp. $337-$ 345, 2009. doi:10.1016/j.medengphy.2008.06.005

[8] L. Yanga, M. Zhaoa, C. Penga, X. Hub, H. Fengc, Z. Ji, "Waveform descriptor for pulse onset detection of intracranial pressure signal", Med. Eng. \& Phys., vol. 34, pp. 179-186, 2012. doi:10.1016/j.medengphy.2011.07.008

[9] E. Kazanavicius, R. Gircys, A. Vrubliauskas, S. Lugin, "Mathematical methods for determining the foot point of the arterial pulse wave and evaluation of proposed methods", Information Technology and Control, vol. 34, no. 1, pp. 29-36, 2005.

[10] A. Fanelli, T. Heldt, "Signal quality quantification and waveform reconstruction of arterial blood pressure recordings", in Conf. Proc. IEEE Eng. Med. Biol. Soc. 2014, pp. 2233-6. doi:10.1109/EMBC. 2014.6944063

[11] P. Augustyniak, "Recovering The Precise Heart Rate From Sparsely Sampled Electrocardiograms", in Computers in Medicine, Łódź 23 25.09.1999, pp. 59-65

[12] K. Lan, J. W. Jorgenson, "A hybrid of exponential and gaussian functions as a simple model of asymmetric chromatographic peaks", Journal of Chromatography A, vol. 915, pp. 1-13, 2001. doi:10.1016/S00219673(01)00594-5

[13] A. Golubev, "Exponentially modified Gaussian (EMG) relevance to distributions related to cell proliferation and differentiation", Journal of Theoretical Biology, vol. 262, pp. 257-266, 2010. doi:10.1016/j.jtbi.2009.10.005

[14] A. Poliński, J. Kot, A. Meresta "Analysis of correlation between heart rate and blood pressure", Federated Conference on Computer Science and Information Systems (FedCSIS), pp. 417-420, 2011

[15] M. Kaczmarek, A. Bujnowski, J. Wtorek, A. Poliński, "Multimodal Platform for Continuous Monitoring of the Elderly and Disabled", J. Med. Imaging Health Inform., vol. 2, no. 1, pp. 56-63, March 2012 doi: http://dx.doi.org/10.1166/jmihi.2012.1061

[16] J. Wtorek, A. Bujnowski, J. Rumiński, A. Poliński, M. Kaczmarek, A Nowakowski, "Assessment of Cardiovascular Risk in Assisted Living", Metrol. Meas. Syst. vol. 19, issue 2, pp. 231-244, May 2012. doi: 10.2478/v10178-012-0020-0

[17] A. Bujnowski, J. Rumiński, P. Przystup, K. Czuszyński,T. Kocejko, "Self Diagnostics Using Smart Glasses-preliminary study", 9th International Conference on Human System Interaction (HSI2016), pp. 511-517, DOI: $10.1109 /$ HSI.2016.7529682

[18] A. Bujnowski, J. Rumiński, M. Kaczmarek, K. Czuszyński, P. Przystup, "Cardiovascular data analysis using electronic wearable eyeglassespreliminary study", Federated Conference on Computer Science and Information Systems (FedCSIS), pp. 1409-1412, 2016.

[19] J. Pan and W. J. Tompkins, "A Real-Time QRS Detection AlgorithmA Real-Time QRS Detection Algorithm" IEEE Trans. on Biomed. Eng. , vol. 32, no. 3, pp. 230-236 March 1985. doi: 10.1109/TBME.1985.325532

[20] A. L. Goldberger, L. A. N. Amaral, L. Glass, J. M. Hausdorff, P. Ch. Ivanov, R. G. Mark, J. E. Mietus, G. B. Moody, C-K. Peng, H. E. Stanley, "PhysioBank, PhysioToolkit, and PhysioNet: Components of a New Research Resource for Complex Physiologic Signals" Circulation 101(23):e215-e220 [Circulation Electronic Pages; http://circ.ahajournals.org/cgi/content/full/101/23/e215]; 2000 (June 13). 